\title{
Refractive-Index Puzzle Explained
}

\section{A new theory explains the lack of variation in the refractive indices of atomic gases.}

By Katherine Wright

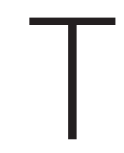
he theories of reflection and refraction are textbook equations taught to students in introductory optics classes. But delve deeper into these theories, and a puzzle pops up: in certain limits, the equations fail. Francesco Andreoli of the Institute of Photonic Sciences, Spain, and colleagues now explain why [1].

The refractive index describes how a material deflects light. The parameter has a value of order 1 for most materials, ranging from around 4 for germanium to slightly over 1 for air. Increasing the atomic density of the material, such that a dilute gas transitions to a dense solid, has little effect on the refractive index-contrary to textbook predictions. Those predictions anticipate a huge increase in a material's refractive index with increasing density because isolated atoms respond to light very strongly.

The team shows that this conflict arises because of how textbook models account for the positions and interactions of atoms in dilute gases. Those models assume that the positions

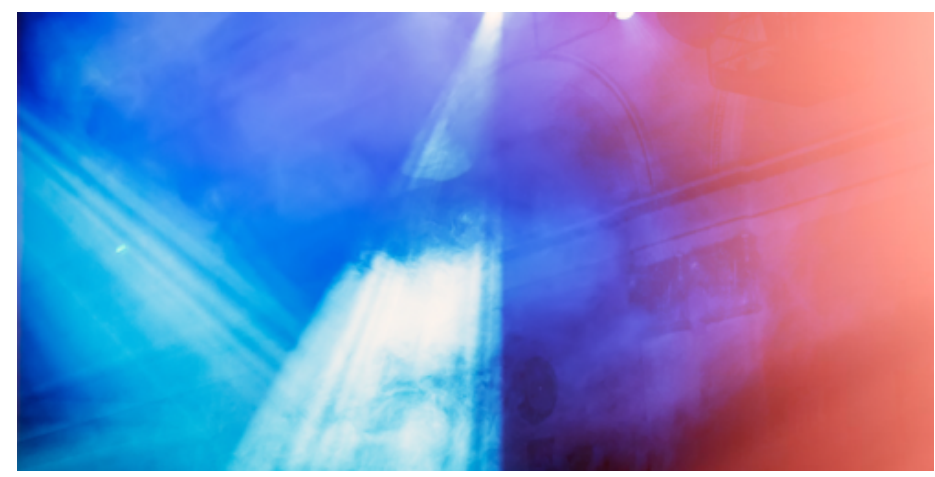

Credit: Aphotostudio/stock.adobe.com don't matter. But Andreoli and colleagues find the opposite. They show that neighboring atoms in dilute gases can strongly interact with each other through short-ranged optical fields. And these interactions can shift the resonant frequency of the atoms such that they no longer strongly deflect light. That shift sets a maximum on the refractive index that is independent of density and leads to the order 1 value consistent with most materials.

As to why this puzzle has remained unsolved for so long, Andreoli says that he, at least, hadn't realized that this problem existed, because of the way the subject is taught. It's exciting to find and solve an issue with textbooks, he adds.

Katherine Wright is a Senior Editor for Physics.

\section{REFERENCES}

1. F. Andreoli et al., "Maximum refractive index of an atomic medium," Phys. Rev. X 11, 011026 (2021). 